Article

\title{
Global Cognitive Functioning versus Controlled Functioning throughout the Stages of Development
}

\author{
Isabel Maria Introzzi ${ }^{1}$, María Marta Richard's ${ }^{1}$, Ana García-Coni ${ }^{1}$, Yesica Aydmune ${ }^{1}$, \\ Florencia Stelzer ${ }^{1}$, Lorena Canet-Juric ${ }^{1}$, Eliana Vanesa Zamora ${ }^{1} \mathbb{D}$, María Laura Andrés ${ }^{1}$, \\ María Fernanda López-Ramón ${ }^{2}$ and Esperanza Navarro-Pardo $2, *$ D
}

1 Instituto de Psicología Básica, Aplicada y Tecnología (IPSIBAT), Universidad Nacional de Mar del Plata (UNMDP)—Consejo Nacional de Investigaciones Científicas y Técnicas (CONICET), Mar del Plata B7602AYJ, Argentina; iintrozzi@mdp.edu.ar (I.M.I.); mrichards@mdp.edu.ar (M.M.R.); anagc@conicet.gov.ar (A.G.-C.); yesicaaydmune@conicet.gov.ar (Y.A.); florenciastelzer@conicet.gov.ar (F.S.); canetjuric@mdp.edu.ar (L.C.-J.); eliana.zamora@conicet.gov.ar (E.V.Z.); mlandres@mdp.edu.ar (M.L.A.)

2 Department of Developmental and Educational Psychology, Faculty of Psychology, Universitat de València, 46010 Valencia, Spain; m.fernanda.lopez@uv.es

* Correspondence: esperanza.navarro@uv.es

Received: 12 October 2020; Accepted: 24 November 2020; Published: 26 November 2020

\begin{abstract}
According to the All or None Hypothesis (Diamond, 2009), the cognitive system can operate in a global manner that is not very discriminate or in a more discriminate mode that demands greater precision, control, and cognitive effort. There are five corollaries to this hypothesis that describe, in an operative way, the conditions under which the controlled mode of functioning in the cognitive domain can be activated and thus tested. Given the impact this theory has generated and the absence of studies analyzing the corollaries in a collective and systematic way at different stages of development, this study was proposed, first of all, to test three of these corollaries in children, adolescents and adults and, secondly, to analyze the changes in the controlled mode of functioning during these three stages of development. To this end, the Fingers Task, a modified version of Arrows Task (with two rules: response ipsilateral where the stimulus is presented, symmetry; and response contralateral, asymmetry), was administered to a sample of 123 participants ( 43 children, 44 adolescents, and 36 adults). In general, the results verify the corollaries and identify the changes that the controlled mode of functioning experiences at different stages of development.
\end{abstract}

Keywords: human development; child; adolescent; adult; cognition; mental processes

\section{Introduction}

People can operate- process information from the environment and respond to it-in a more controlled or more automatic way, depending on their possibilities and/or situational requirements. Of these two ways of functioning, the global one, which is activated instantly, appears as the most frequent and natural, both in children and adults [1-4]. We can find evidence of this in the creation of the first groups of neural connections. These connections are characterized by being global and nonspecific. For this reason, the vertiginous growth of neural connections in the first years of life is replaced later in development by a process of elimination-pruning — which endows the cognitive system with greater specificity and efficiency. This process culminates in the transition from adolescence to adulthood [5-7].

This dual processing way of the cognitive system led Diamond [2] to propose the "All or None Hypothesis", which systematizes this duality in terms of a global mode of functioning-which would be activated by default-and a more selective, controlled and discriminated mode-which would be activated only if necessary. The fundamental difference lies on the fact that the global mode requires 
less effort and resources than the selective mode, hence the most efficient and fastest way to operate is indeed through this default mode, as long as it allows us to function correctly in the situation. This is the case of tasks and situations that involve strongly ingrained behaviors, whether due to prior learning, reinforcement, or inheritance.

However, when the situation requires more specific responses and voluntary control, it is necessary to deactivate that predetermined mode - the global mode - that is, to inhibit the initial and preponderant reactions, to give rise to a more selective mode that allows us to act appropriately in that situation. This deactivation has a cost, which is subjectively experienced as a cognitive effort and objectively manifested as an increase in Reaction Time (RT), that is, a slower performance. Sometimes-especially in the case of children-an increase in errors also occurs.

This differentiation between a global mode and a selective mode, which supposes a dual logic, finds its correlation in the classical distinction between automatic and deliberate attention, and also between ascending/descending, endogenous/exogenous, implicit/explicit, unconscious/conscious, Type I/Type II, or heuristic/analytic processes [3,4,8].

The aforementioned "All or Nothing Hypothesis" [2] comprises five corollaries that expose the duality of the cognitive system in various domains and, therefore, allow grouping a large and heterogeneous amount of empirical results. In a previous study with adult participants [9], evidence was found in favor of three of the five corollaries proposed by Diamond [2]. According to that study, the global and undifferentiated mode of functioning is activated when fast and unspecific responses are required. However, when the context demands a greater response control, the discriminated mode shows up, replacing the global one, with the consequent manifestation of greater effort and cognitive cost. What the present study brings about, as a continuation of the previous one [9], is the analysis of the corollaries on which the All or Nothing Hypothesis are based, and at different stages of the life course.

So far, there are no studies to our knowledge that have contrasted several of these corollaries using the same experimental task and in different evolutionary stages. On the other hand, there is also insufficient evidence about the distinctive changes and operational characteristics of the selective or controlled processing mode in adolescence. In this sense, much of the studies carried out on the development of the cognitive control function have focused on childhood, adulthood, or old age [10]. The interest and the amount of work destined to analyze these types of changes in adolescence seems substantially less compared to the rest of the developmental stages, probably due to the belief during the $60 \mathrm{~s}-70 \mathrm{~s}^{\prime}$ that stated that there were no substantial changes during adolescence in frontal lobes, being the brain areas on which cognitive or executive control depends [5,11]. However, these ideas are being revised due to a set of relevant neurosciences findings [12,13] and nowadays, adolescence is conceived as a sensitive period of development characterized by the presence of a diverse set of brain, emotional, and cognitive changes. The actual scope encourages further research to analyze these issues. In this sense, this paper constitutes a clear contribution to this research area not only because it accurately describes the specific processing mode of selective and comprehensive functioning in adolescence, but also because it analyzes the differences and similarities in comparison to other stages of development such as childhood and adulthood.

In sum, this study aims to analyze three corollaries of the aforementioned "All or none Hypothesis", in three stages of development: childhood, adolescence, and adulthood. Thus, the stability and change in the controlled mode of functioning during these stages are also explored. For this reason, the next section will begin by exhaustively defining and operationalizing the corollaries.

\section{Materials and Methods}

\subsection{Corollaries}

The first corollary states that when something must be changed in relation to a certain activity, it is regularly easier to change everything or change nothing (global) than to make a partial change. 
This effect has been documented in both adults and children [14-16] and can be tested via change tasks, analyzing conditions of no change, total change, and partial change. Thus, in a study [17] that children from 4 to 13 years old and young adults participated in, this corollary was confirmed throughout the age spectrum, since fewer errors were made in the total change than in the partial change condition. However, in terms of response time, only children over 9 years old and young adults performed a total change faster than a partial change. These results suggest that after this age a partial change demands a more discriminated and controlled process than a total change, which would imply a lower level of cognitive control.

In summary, some authors [17] suggest that change is essentially difficult and is an example of top-down executive control, since it generally cannot be done in an "automatic way". In addition, alternating between tasks implies a slow progressive development. This was confirmed in the aforementioned study, since even at the age of 10 years the correct answers did not exceed $80 \%$, and at 13 the performances still did not reach adult levels. In fact, much of the cost reduction of exercising cognitive flexibility happens after that age. In this regard, there is a great deal of research that convincingly demonstrates systematic age-related improvements in executive control during adolescence $[7,18]$.

The second corollary expresses that it is easier to consider various attributes of a stimulus than only one of its properties. As Diamond [2,19] explains, indeed it is often challenging to ignore irrelevant properties of an attended stimulus. From this statement we infer that not paying attention or ignoring the irrelevant properties of a stimulus implies a controlled mode, whereas taking into account multiple salient attributes of a stimulus may require a global mode of processing.

This corollary is evidenced, for example, on Simon effect, which describes the human preference to respond to the location where the stimulus is presented [19-22]. Therefore, in tasks that demand an ipsilateral response to the stimulus (stimulus/response site congruence), people present more accurate and faster responses than when a contralateral response to the stimulus is required (stimulus/response site incongruence) [21,23].

In other words, in the Simon task, participants must respond to the stimulus identity (relevant property) and ignore its location (irrelevant property). The cost of doing that is reflected in the increase of RT and mistakes in the condition in which stimulus location must be ignored and selective attention to its identity (relevant information) must be paid. Although the Simon effect has been reported in both children [17] and adults [19,24], its presence is much more pronounced in young children $[17,25]$. However, these results have not been replicated in other studies [26].

Finally, the third corollary theorizes that it is easier to inhibit a dominant response most of the time (global mode) than doing it sometimes. In this respect, inhibiting a dominant response, as indicated in the second corollary, requires effort, but when that inhibition must be maintained consistently (global mode), not as much effort is required. It is much more demanding constantly alternating between inhibiting a dominant response sometimes and giving the dominant response other times. That is, if the same mindset is maintained, the effort invested will be less $[17,27,28]$. Similarly, Davidson et al. [17] found that inhibiting the dominant response only sometimes, as opposed to inhibiting it consistently across all trials, came at the expense of speed and accuracy at all ages, even in adult groups were they found a similar switching cost, except in the accuracy index, in which adults showed less cost in comparison to younger children.

The difficulty of the most challenging condition (incongruent) is miscalculated when that response is always called for; in other words, continually responding on the opposite side the stimulus reduces the difficulty of the response itself because participants enter into a "response rhythm". Likewise, the advantage of the easier (congruent) condition is underestimated when participants must alternate between that response and the incongruent one; this happens because people's performance tends to slow down in contexts of change [17].

As it was mentioned before, the All-or-None Hypothesis mode [2] contributes to a general setting that allows articulating a series of highly heterogeneous findings related to the cognitive 
functioning seen in different age groups. Nonetheless, most of the scientific evidence has been focused on a particular developmental stage and in some corollaries $[9,29,30]$. Up to date, there are no studies to our knowledge that have attempted to systematically and simultaneously analyze whether these corollaries resulting from this general setting are held among age groups. Therefore, the present study aims to, firstly, verify three of the All or None Hypothesis' corollaries in children, adolescents, and adults, and, secondly, analyze the effects of development on the controlled mode of cognitive functioning. In summary, there are no studies to our knowledge that have analyzed these corollaries $(1,2$, and 3$)$ simultaneously, using only one experimental task that analyzes an overall contrast and comparing the performance of the same participant in diverse conditions that demands different modes of cognitive functioning and been assessed in different stages of development.

\subsection{Participants}

The sample consisted of 123 participants of both sexes with ages ranging from 9 to 44 years. The sample was divided into three groups: Group 1 (G1), composed of 43 children (22 males and 21 females), 9 and 10 years old $(\mathrm{M}=9.37, \mathrm{SD}=0.48)$; Group $2(\mathrm{G} 2)$, consisting of 44 adolescents (20 males and 24 females), $12-19$ years old ( $M=16.0, \mathrm{SD}=2.2$ ); Group 3 (G3), composed of 36 adults (15 males and 21 females), $20-44$ years old $(M=31.35, S D=8.2)$. Participants from all age groups are mainly from middle socioeconomic status-Group 1: $16.4 \%$ low; $60.2 \%$ middle; $23.4 \%$ high; Group 2: $11.3 \%$ low; $61.6 \%$ middle; $25.1 \%$ high; Group 3: $10.2 \%$ low; $66.2 \%$ middle; $23.6 \%$ high [31].

The selection of children and adolescents was carried out by simple random sampling with replacement from a total sample of 150 students from two public schools (Mar del Plata, Argentina). The study included nonrepeating students who were not in psychological and/or psychiatric treatment at the time of the evaluation and had no history of learning disorders or developmental disorders as reported by teachers. The adult group included participants who had completed high school, were not in psychiatric and/or neurological treatment and did not present any disorder or condition suspected of interfering with the administration of the instrument (severe motor, visual, or mental disorders).

\subsection{Instruments}

For the analysis of the three corollaries, a version of the arrow task proposed in Davidson et al. [13], a classic task switching instrument, was used because it demands the ability to change quickly between different types of rules and responses. This instrument is included in a platform called Cognitive Self-Regulation Tasks [32] under the name the Fingers Task (since images of a hand pointing with the index finger are used as stimuli instead of arrows).

The task consisted of three experimental blocks that were presented in the following sequence: Congruent Block (CB), Incongruent Block (IB), and Mixed Block (MB). Prior to each experimental block, a practice block of eight trials appears. Each practice block was the same as the corresponding experimental block, the difference being that it was composed of fewer trials and that the performance therein was not used for the calculation of the performance indexes. If the participant fails to respond correctly in $80 \%$ of the practice block trials, the experimental block (which is used to record performance measures) will not begin, and the practice block must be administered again until this threshold is met.

The CB was the first experimental block. Prior to its administration, the practice block was presented comprising eight randomized congruent trials. In this block, a hand with a finger pointing appears pointing down on the left or right side of the screen, then the participant must press the key ipsilateral to the site where the stimulus is presented - " $\mathrm{Z}$ " or " $\mathrm{M}$ "— on the keyboard. There was a certain symmetry, because the stimulus and the key are in the same lateral. Previous studies showed that there is a prepotent tendency to respond in the same location as the stimulus. Thus, in this condition, shorter RTs and greater precision are expected $[17,19,20]$. In the experimental block, 10 stimuli distributed randomly were presented on the left side and 10 were presented on the right side of the screen. 
When the CB was completed, the IB was presented with the corresponding practice block, both being composed only by incongruent trials. In this case, the stimulus consisted of a hand with its index finger pointing in a diagonal direction (at a $45^{\circ}$ angle) to the opposite side in which it is presented. Thus if the hand appears on the right side of the screen, it points to the contralateral response site and the participant must therefore press the letter " $\mathrm{Z}$ "; conversely, if it appears on the left side, the finger points to the contralateral response site and the participant must press the " $\mathrm{M}$ " key. In summary, the diagonal-pointing hand always points to the opposite side, indicating that the key that is contralateral to the side where the stimulus is presented must be pressed. Here, there is a certain asymmetry between the location of the stimulus and the site of the key and the prepotent tendency to respond in the same location as the stimulus must be inhibited. Thus, in this condition, people are slower and make more errors, because cognitive control and effort is implicated $[17,19,20]$. The practice block consisted of eight trials and the experimental block had 20 trials (10 trials where the stimulus was presented on the right side of the screen and in the other 10 trials was presented on the left side). In both cases the stimuli were distributed randomly.

Following the IB, the MB) was presented with its practice block of eight trials. In both congruent stimuli (hand pointing straight down) and incongruent stimuli (hand pointing to the opposite side) appeared, distributed randomly. The practice block consisted of four congruent and four incongruent trials (half of the stimuli of each type were presented on the left side of the screen and the other half were presented on the right side). The MB consisted of 40 trials: 20 congruent and 20 incongruent. The stimuli were distributed randomly respecting the following conditions: 20 stimuli on the right side (10 congruent and 10 incongruent) and 20 stimuli on the left side (10 congruent and 10 incongruent).

The MB is a classic task-switching behavioral task in which the participant typically must switch quickly and effectively between two conflicting rules (pressing the same side or pressing the opposite side). For it, the achievement of the correct responses on the mixed block demands a continuous configuration and reconfiguration of actions $[15,17,33,34]$. Figure 1 shows the three main blocks.

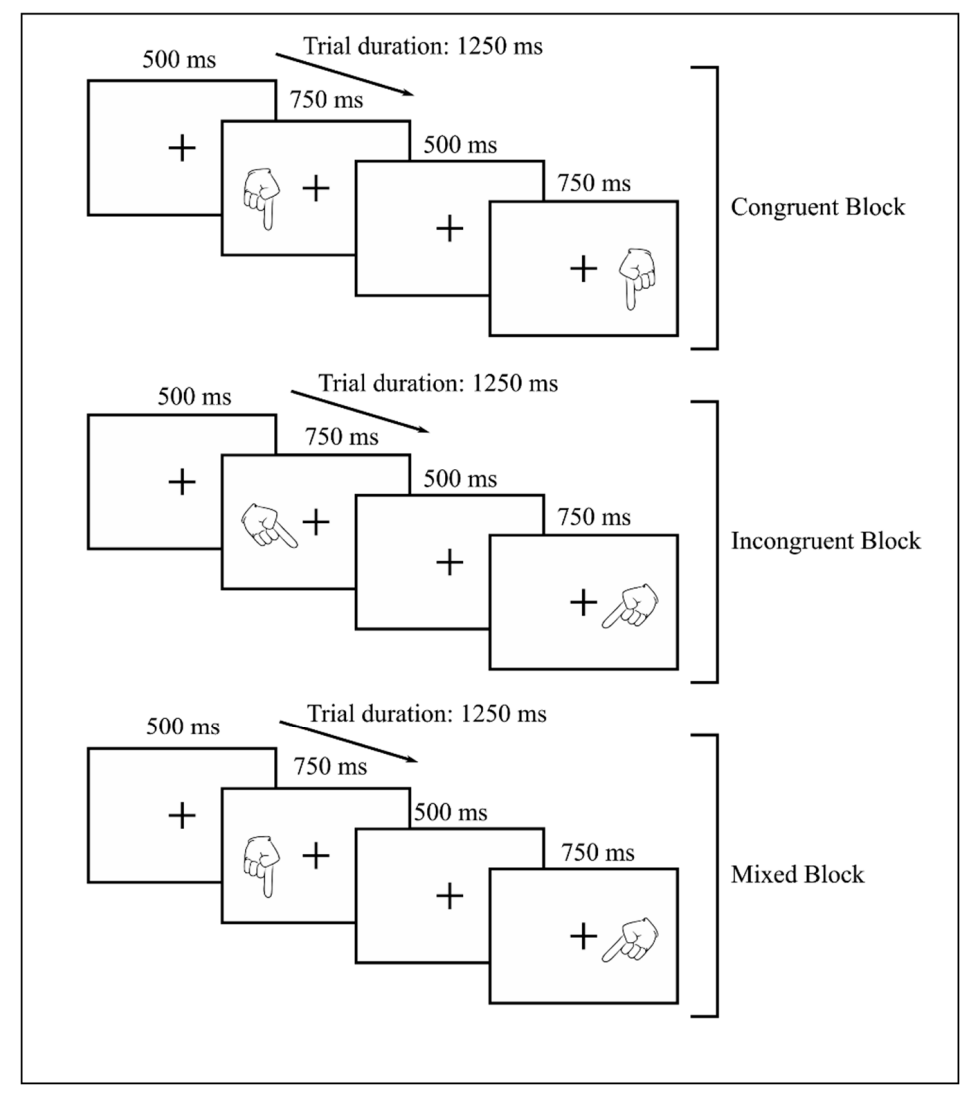

Figure 1. Examples of congruent and incongruent trials in all blocks from the Finger Task. 
In each block, the following sequence was repeated: first a fixation point appears (a cross) in the center of the screen and stays throughout the block. Next, the stimuli appeared sequentially on the left or right side of the fixation point an equidistant with a stimulus interval of $500 \mathrm{~ms}$. Each stimulus remained on the screen for a time of $750 \mathrm{~ms}$, and during this period the participant had to respond.

The measurement of performance on the experimental blocks included a set of basic performance measures: (a) the average percentage of correct responses, (b) the average RT for each trial type (congruent and incongruent), and (c) the amount of anticipatory responses (responses emitted in $200 \mathrm{~ms}$ or less).

\subsection{Operative Description of Corollaries, Hypotheses, and Expected Results}

In the operative description of each corollary, two aspects were included: one related to the contrast of its basic situations in each age group (corollaries contrast) and the second associated to the analysis through the age/vital stage groups considered (corollaries development).

Corollary 1. When something must be changed, it is always easier to change everything or change nothing than change just one aspect.

As it was mentioned in the Section 1, this corollary was analyzed through three conditions: (a) no change condition; (b) partial change condition; (c) total change condition. Summing-up, according to the All or Nothing Hypothesis, while conditions (a) and (c) represent a less discriminated way of acting (b) represents a more specific mental operation, which therefore requires cognitive control and effort.

The mixed block allows us to generate a number of indexes that reflect the performance in conditions, which allows us to contrast this corollary. The no change condition (a) is obtained from the average RT and the number of correct answers (ACC) in trials with no rule change or response site; that is, the condition in which is repeated the same rule (congruent or incongruent) and the same response location (" $\mathrm{Z}$ " or " $\mathrm{M}$ " key) as the trial preceding it. Both indexes reflect an achievement-in trials preceded by trials - that is precisely the same in terms of both the rule and the response location. On the other hand, condition (c) represents total change. This condition includes the average RT and the ACC in trials where the rule and response site change compared to the preceding trial. Finally, the partial change condition (b) is analyzed based on the average RT and the ACC in trials that require a rule change but not a response location change compared to the preceding trial (Different Rule Same Site-DRSS) as well as in trials that requires the same rule but a different response site (Same Rule Different Site-SRDS).

In summary, according to the All or None Hypothesis, partial change indexes should reflect a higher RT and a lower percentage of correct answers than total change and no change indexes (corollaries contrast). Additionally, this hypothesis implies the existence of an improvement in the controlled mode due to the effect of cognitive development; hence a significant decrease in RT and an increase in accuracy (ACC) is expected based on participants' developmental stage (children, adolescents, and adults) (corollaries development).

Corollary 2. It is easier to consider considerable salient properties of a stimulus than only one of its properties.

According to this corollary, it is often laborious to ignore irrelevant properties of a stimulus [2], and this is why this corollary is related to the controlled processing.

The task used is sustained by the Simon experimental effect described in Section 1. In this task, the relevant attribute is the direction to which the finger is actually pointing (a hand with a finger pointing down or a hand with a finger pointing to a diagonal direction) and the irrelevant attribute is the location (the left or right side of the screen). Therefore, the participant must pay attention to the stimulus identity (relevant property) and not to its location (irrelevant property). However, as the Simon effect shows, the stimulus identity is less salient and prominent than the irrelevant attribute (stimulus location), and the connection to the response site is weaker and it must be established in 
the course of a few trials. On the contrary, the irrelevant attribute is more salient and naturally-and biologically-connects to the response site.

Thus, according to the aforementioned in corollary 2, participants are expected to obtain lower $\mathrm{RT}$ and greater ACC in trials where the stimulus identity is consistent with the location (congruent trials), and where the global and automatic processing mode is sufficient, than in those that must focus their attention on less salient properties (incongruent trials), i.e., respond in a discriminate and controlled manner (corollaries contrast). Furthermore, due to the developmental effect on the controlled processing mode, there should be age-related improvement in incongruent conditions (corollaries development).

Corollary 3. It is easier to inhibit a proponent response all the time than to inhibit it only at times.

This corollary states that although inhibition implies cognitive effort and control, it is easier to inhibit the response all the time than to do so only on certain occasions. In other words, it establishes that it is easier to always act the same way than to sometimes act in a certain way and at other times another way.

In the Finger Task, inhibition is involved in the incongruent block and the mixed block, as both include incongruent trials that force the participant to inhibit the dominant response (ipsilateral response). The great difference between these blocks is the proportion of incongruent trials presented on each. In this way, while in the incongruent block $100 \%$ of the trials are incongruent, in the mixed block only $50 \%$ of the trials are incongruent. This characteristic allows us to discriminate between two conditions: condition IB, in which the participant must inhibit the prevailing response in all the tests, and condition $\mathrm{MB}$, in which the participant must only inhibit this response in $50 \%$ of the trials (i.e., sometimes they must inhibit and sometimes they have not).

According to the All or None Hypothesis, although inhibition is considered a process that requires effort and control, condition IB is less complex than condition MB, which implies inhibition only in certain trials. Therefore, in condition MB the tendency to respond in the same way must always be taken into account, as the activity also includes congruent trials requiring another response (no inhibitory trials). This should translate to a significantly better performance in the purely incongruent block compared to the mixed block one (block effect) and a better performance in trials that are preceded by a same type trial (congruent or incongruent) than in those that are preceded by a different kind trial (congruent or incongruent) in the mixed block (change effect) (corollaries contrast).

Similarly, the developmental effect on the controlled mode of processing should manifest itself in better performance with age in mixed block trials that involve a rule change (corollary development).

\subsection{Procedures}

Participation in the study was voluntary and authorized by informed consent, and in the case of minors, the informed consent of their parents or guardians were additionally assessed. The informed consent form explained the objectives of the study and described the task to be administered. It was also made clear that the data were confidential and that the results would only be used for research purposes. Our research team was authorized by the ethics committee of the National University of Mar del Plata for the study of cognitive flexibility and application of the task at different stages of development. Additionally, it is worth mentioning that the current study strictly followed the guidelines for ethical behavior in Social Sciences and Humanities given by the National Council for Scientific Research and Techniques of Argentina [35], the criteria recommended by the American Psychological Association [36] for activities destined to obtain knowledge about psychological processes in human beings, and the ethical principles for research with human beings stipulated by the Declaration of Helsinki [37].

In the case of children and adolescents, the task was administered individually in the schools' classrooms used for this purpose. As it was a computerized behavioral task, laptops were used. In the 
case of adults, administration was also performed individually with laptops in a quiet place without interruptions. The duration of administering the task was approximately $10 \mathrm{~min}$.

\subsection{Data Analysis}

Eleven participants were excluded because they presented a number of anticipatory responses greater than 2 SD in at least one block of the Fingers Task. Based on these criteria, we excluded cases that presented a total of 10 or more anticipatory responses in CB and IB), and a total of six or more in the MB. The decision to exclude these trials was based on the fact that this type of response is considered too fast $(<200 \mathrm{~ms})$ to be qualified as a response to a stimulus [17]. For the calculation of RT-based measures, only correct answers were included.

Before the statistical tests were applied, the trade off effect, which refers to the response style adopted by the participants, was also analyzed. Basically, this effect indicates whether during the performance of the task the participant chose to take more time to respond, sacrificing speed in order to maintain accurate performance or vice versa [38]. The absence of correlations between RT and response accuracy in the different measures allowed us to discard the effect and base the analysis of RT and ACC as independent measures of performance. The same process was undertaken for the analysis of each corollary.

In order to analyze the differences between the controlled and global processing mode, a repeated measures ANOVA was applied that included the measures described for each corollary. Likewise, to evaluate the developmental effect on the controlled mode (Change), a $3 \times 4$ GLM (Group Factor: children-adolescents-adults/Change condition: SRSS-DRSS-SRDS-DRDS) was applied. Based on the results of the GLM, post hoc comparisons were made to identify the groups between which differences were found. Prior to the comparison of each corollary, the normality assumption was tested for most measures (Shapiro-Wilk $p>0.05$ ). In the cases where violation of the sphericity assumption was observed, the Greenhouse-Geisser correction was used. In cases in which the homoscedasticity assumption was not tested, the comparison method recommended by Games Howell was used; in the rest of the comparisons the Bonferroni method was applied. In addition, the interaction effects between the Change condition and the Age group were analyzed.

\section{Results}

\subsection{Corollary 1}

When something must be changed in relation to a certain activity, it is easier to change everything or change nothing, than to change a single aspect-partial change (corollary contrast). In addition, there should be age-related improvement in the controlled mode due to the effect of development; hence a significant decrease in RT (faster) and in response accuracy (greater) is expected based on participants' developmental stage (corollary development).

The descriptive statistics in ACC and RT for the main independent variables discriminated by Age group in the different conditions of change are shown in Table 1.

Table 1. Mean accuracy and mean Reaction Time (RT) for the different change conditions by Age group.

\begin{tabular}{ccccccccc}
\hline \multirow{2}{*}{ Group } & \multicolumn{2}{c}{ Absence of Change } & \multicolumn{2}{c}{$\begin{array}{c}\text { Partial Change } \\
\text { SRDS }\end{array}$} & \multicolumn{2}{c}{$\begin{array}{c}\text { Partial Change } \\
\text { DRSS }\end{array}$} & \multicolumn{2}{c}{ Total Change } \\
\cline { 2 - 9 } & RT & Accuracy & RT & Accuracy & RT & Accuracy & RT & Accuracy \\
\cline { 2 - 9 } G1 & $633(104)$ & $94.8(10.3)$ & $762(134)$ & $86.2(17.4)$ & $749(121)$ & $86.2(13.2)$ & $853(133)$ & $72.3(20.6)$ \\
G2 & $487(60)$ & $99.3(2.5)$ & $597(109)$ & $95.2(7.9)$ & $572(76)$ & $89.0(11.8)$ & $626(122)$ & $89.1(10.2)$ \\
G3 & $540(81)$ & $92.2(11.8)$ & $639(98)$ & $96.9(10.3)$ & $694(114)$ & $91.8(12.3)$ & $639(86)$ & $95.6(9.7)$ \\
\hline
\end{tabular}

Note: G1 = Child group (M = 9.37, 9-10 years); G2 = Adolescent group; $(\mathrm{M}=16.0$, 12-19 years); G3 = Adult group $(\mathrm{M}=31.35,20-44$ years $) ; \mathrm{RT}=$ Reaction Time; SRDS = Same Rule Different Site; DRSS = Different Rule Same Site. 
Change condition showed a significant within-subjects effect on both RT with $\mathrm{F}(2.59,311.305)=146.345, p<0.001, \eta 2=0.55$, and accuracy with $\mathrm{F}(2.725,326.96)=33.35, p<0.001$, $\eta 2=0.22)$. Age group showed a significant between-subjects effect both on RT with $\mathrm{F}(2,120)=41.5$, $p<0.001, \eta 2=0.41$, and accuracy with $\mathrm{F}(2,120)=14.34, p<0.001, \eta 2=0.19$. Additionally, a significant age group $\mathrm{x}$ condition interaction was observed in both $\mathrm{RT}$ with $\mathrm{F}(5.19,311.305)=14.64, p<0.001$, $\eta 2=0.2$, and accuracy with $\mathrm{F}(5.45,326.96)=10.435, p<0.001, \eta 2=0.15$, as shown in Figure 2.
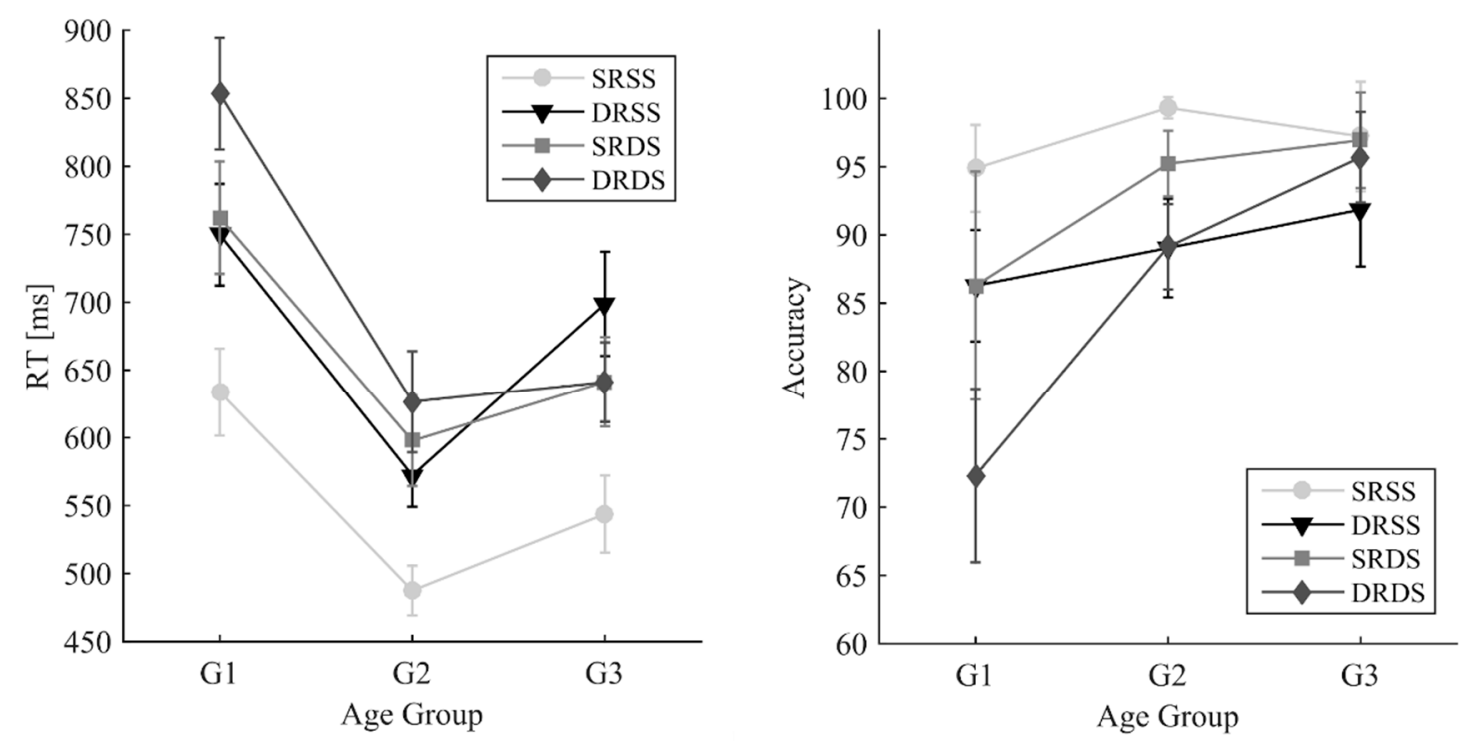

Figure 2. Reaction Time (RT) and Accuracy for the different conditions and Age Group. SRSS = Same Rule Same Site; SRDS = Same Rule Different Site; DRSS = Different Rule Same Site; DRDS = Different Rule Different Site. The bars represent the standard error.

The interaction was analyzed by (1) calculating the effect of condition for each age group (corollary contrast), and also by (2) calculating the effect of age group for each condition (corollary development). For the first analysis, the results showed that a significant effect of condition was observed on RT with $\mathrm{F}(3,126)=72.54, p<0.001, \eta 2=0.63$, in children $(\mathrm{G} 1) ; \mathrm{F}(1.99,85.58)=40.17, p<0.001, \eta 2=0.48$, in adolescents (G2); $F(3,105)=78.65, p<0.001, \eta 2=0.69$, in adults (G3). A significant effect on accuracy was also observed with $\mathrm{F}(2.58,108.515)=24.895, p<0.001, \eta 2=0.37$, in $\mathrm{G} 1 ; \mathrm{F}(2.06,88.74)=16.235$, $p<0.001, \eta 2=0.27$, in G2; $\mathrm{F}(1.69,59.01)=5.64, p=0.008, \eta 2=0.14$, in G3. Pairwise comparisons between conditions for each group are shown in Table 2.

Table 2. Comparisons of accuracy and Reaction Time (RT) among the different change conditions by age group.

\begin{tabular}{|c|c|c|c|c|c|c|c|}
\hline \multirow{2}{*}{\multicolumn{3}{|c|}{ Comparisons }} & \multicolumn{2}{|c|}{ G1 } & \multicolumn{2}{|c|}{ G2 } & \multirow{2}{*}{$\begin{array}{l}\text { G3 } \\
\text { RT }\end{array}$} \\
\hline & & & Accuracy & RT & Accuracy & RT & \\
\hline \multirow{3}{*}{ Absence of change } & \multirow{3}{*}{ vs. } & DRSS & $p<0.001$ & $p<0.001$ & $p<0.001$ & $p<0.001$ & $p<0.001$ \\
\hline & & SRDS & $p=0.01$ & $p<0.001$ & $p=0.002$ & $p<0.001$ & $p<0.001$ \\
\hline & & Total change & $p<0.001$ & $p<0.001$ & $p<0.001$ & $p<0.001$ & $p<0.001$ \\
\hline \multirow{2}{*}{ Partial change DRSS } & \multirow{2}{*}{ vs. } & SRDS & ns & ns & $p=0.03$ & ns & $p<0.001$ \\
\hline & & Total Change & $p<0.001$ & $p<0.001$ & ns & $p=0.01$ & $p<0.001$ \\
\hline Partial change SRDS & vs. & Total Change & $p<0.001$ & $p<0.001$ & $p=0.001$ & $p=0.01$ & ns \\
\hline
\end{tabular}

Note: The comparisons for accuracy are not shown in G3 since there was no change effect in this group. $\mathrm{RT}=$ Response time; SRDS = Same Rule Different Site; DRSS = Different Rule Same Site; ns = not significant.

For the second analysis, results showed that a significant effect of age group was observed on RT with $\mathrm{F}(2,120)=32.83, p<0.001, \eta 2=0.35$, for the SRSS condition; $\mathrm{F}(2,120)=23.26, p<0.001, \eta 2=0.28$, for the SRDS condition; $\mathrm{F}(2,120)=32.91, p<0.001, \eta 2=0.35$, for the DRSS condition; $\mathrm{F}(2,120)=49.28$, 
$p<0.001, \eta 2=0.45$, for the DRDS condition. A significant effect on accuracy was also observed with $\mathrm{F}(2,120)=8.41, p<0.001, \eta 2=0.12$, for the SRDS condition; with $\mathrm{F}(2,120)=27.32, p<0.001, \eta 2=0.31$, for the DRDS condition. Age group did not show a significant effect on accuracy in the SRSS and DRSS conditions. Pairwise comparisons between Age groups for each condition are shown in Table 3.

Table 3. Differences between groups for the different measures of change.

\begin{tabular}{ccccccccc}
\hline \multirow{2}{*}{ Comparison between Groups } & \multicolumn{2}{c}{ G1 1 G2 } & \multicolumn{2}{c}{ G2 $\neq$ G3 } & \multicolumn{2}{c}{ G1 $\neq$ G3 } \\
& & RT & Accuracy & RT & Accuracy & RT & Accuracy \\
\hline No change & SRSS & $p<0.001$ & $\mathrm{~ns}$ & $p=0.01$ & $\mathrm{~ns}$ & $p<0.001$ & $\mathrm{~ns}$ \\
Partial change & SRDS & $p<0.001$ & $p=0.004$ & $\mathrm{~ns}$ & $\mathrm{~ns}$ & $p<0.001$ & $p=0.001$ \\
Total change & DRSS & $p<0.001$ & $\mathrm{~ns}$ & $p<0.001$ & $\mathrm{~ns}$ & $\mathrm{~ns}$ & $\mathrm{~ns}$ \\
& DRDS & $p<0.001$ & $p<0.001$ & $\mathrm{~ns}$ & $\mathrm{~ns}$ & $p<0.001$ & $p<0.001$ \\
\hline
\end{tabular}

Note: G1 = Child group; G2 = Adolescent group; G3 = Adult group; RT = Response time; SRDS = Same Rule Different Site; DRSS = Different Rule Same Site; ns = not significant.

\subsection{Corollary 2}

It is less difficult to consider the salient properties of a stimulus-when the stimulus identity is consistent with its location, in congruent trials-than considering only one of its properties-when the stimulus location is not consistent with its identity, in incongruent trials-(corollary contrast). In addition, due to the developmental effect in the controlled mode of processing, there should be an age-related improvement in incongruent conditions (corollary development).

In order to analyze that all the situations of the corollary contrast were met, a $t$-test was carried out with dependent samples. Data showed the expected effect on all the comparisons between $C B$ and IB (corollary contrast). That is, increased RT and decreased accuracy in IB indicates that targeting the less salient aspects of the stimulus requires more controlled and discriminate processing. This result is not replicated in all the comparisons between congruent trials and incongruent trials of the $\mathrm{MB}$, as only significant differences in RT measurements were obtained in G2 and G3. Regarding the accuracy of response, the effect was not found in any of the groups. Table 4 shows the descriptive statistics and comparisons between the different conditions.

Table 4. Comparisons between different change conditions and trials by Age group.

\begin{tabular}{cccccccccc}
\hline \multirow{2}{*}{ Group } & CB & IB & \multicolumn{7}{c}{ MB } \\
& & M(SD) & M(SD) & T & d & Cong.-Incong. \\
& & M(SD) & M(SD) & T & d \\
\hline \multirow{2}{*}{ G1 } & RT & $411(88)$ & $502(69)$ & $-7.4^{* * *}$ & $-1.15(-0.49)$ & $719(110)$ & $727(116)$ & -0.72 & -0.07 \\
& ACC & $96.2(5.2)$ & $92.5(6.8)$ & $2.9^{* *}$ & $0.72(0.34)$ & $85.7(14.0)$ & $84.0(13.4)$ & 0.78 & $(-0.03)$ \\
G2 & RT & $342(41)$ & $407(55)$ & $-8.1^{* * *}$ & $-1.33(-0.55)$ & $577(80)$ & $572(81)$ & $3.38^{* *}$ & $0.12(0.06)$ \\
& ACC & $98.9(2.0)$ & $94.6(7.6)$ & $3.8^{* * *}$ & $0.77(0.33)$ & $92.1(6.9)$ & $94.3(6.2)$ & -1.7 & $0.06(0.03)$ \\
G3 & RT & $395(93)$ & $497(94)$ & $-7.4^{* * *}$ & -1.09 & $613(87)$ & $640(95)$ & $-3.80^{* * *}$ & $-0.33(-0.16)$ \\
& ACC & $99.0(2.6)$ & $92.9(11.2)$ & $3.5^{* * *}$ & $0.75(0.35)$ & $93.9(4.6)$ & $94.0(15.9)$ & 1.3 & 8.01 \\
\hline
\end{tabular}

Note: $\mathrm{G} 1$ = children group; G2 = adolescent group; G3 = adult group; $\mathrm{M}=$ average; $\mathrm{SD}=$ standard deviation; $\mathrm{ACC}=$ Accuracy; $\mathrm{RT}=$ Response Time; $\mathrm{MB}=$ Mixed Block; $\mathrm{IB}=$ Incongruent Block; Cong. = Congruent trials; Incong. $=$ Incongruent trials. ${ }^{* *} p<0.001{ }^{* *} p<0.01$

With the purpose of analyzing the controlled mode of the developmental hypothesis (corollary development), an ANOVA was performed, resulting in a significant main effect for the Group factor in both IB RTs (F (2) = 22.24; $p<0.001, \eta 2=0.27)$ and in MB incongruent trials $(\mathrm{F}(2)=30.27, p<0.001$, $\eta 2=0.33)$. Regarding the accuracy measures, the effect was found in MB incongruent trials $(\mathrm{F}(2)=9.45$, $p<0.001, \eta 2=0.13$ ). The post hoc comparison methods allowed us to detect among which groups the differences in the different conditions were presented. In general, contrasts showed differences among the different groups, except for children and adults, who had similar IB RTs. The results are summarized in Table 5. 
Table 5. Comparison between IB and MB incongruent trials.

\begin{tabular}{ccccc}
\hline Comparison between Groups & & G1 $\neq \mathbf{G} 2$ & G2 $\neq$ G3 & G1 $\neq$ G3 \\
\hline $\mathrm{IB}^{\mathrm{a}}$ & $\mathrm{RT}$ & $p<0.001$ & $p<0.001$ & $\mathrm{~ns}$ \\
& $\mathrm{RT}$ & $p<0.001$ & $p<0.01$ & $p<0.001$ \\
Incong.- $-\mathrm{MB}^{\mathrm{b}}$ & Accuracy & $p<0.001$ & $\mathrm{~ns}$ & $p<0.001$ \\
\hline
\end{tabular}

Note: $\mathrm{G} 1$ = children group; G2 = adolescent group; G3 = adult group; RT = Response Time; IB = Incongruent Block;

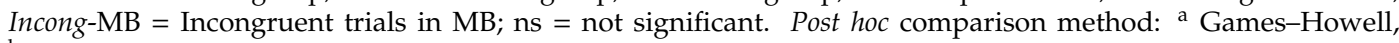

b Bonferroni.

\subsection{Corollary 3}

It is less difficult to inhibit a dominant response all the time than it is to do so only some of the time. This should translate to a significantly better performance in the purely IB compared to the MB-block effect-and a better performance in trials that are preceded by a similar trial than in those that are preceded by a trial of a different type in the MB — change effect-(corollary contrast). The developmental effect on the controlled mode of processing should manifest itself in better performance with age in mixed block trials that involve a rule change (corollary development).

A $t$-test was conducted for related samples to verify the corollary contrast situations. We find the existence of the two expected effects, block effect and change effect, in all three groups and in all RT measures (Table 6). Figure 3 shows the change effect for accuracy and response time. Regarding the accuracy of response, while the change effect was found in all three groups, the block effect was only present in G1. The results are summarized in Table 6.

Table 6. Comparisons between blocks (incongruent and mixed) and between trials with and without rule change (mixed block).

\begin{tabular}{|c|c|c|c|c|c|c|c|c|c|}
\hline \multirow{2}{*}{\multicolumn{2}{|c|}{ Group }} & \multirow[b]{2}{*}{ IB } & \multirow[b]{2}{*}{ MB } & \multicolumn{6}{|c|}{ MB } \\
\hline & & & & & & $\begin{array}{c}\text { Repeat Trials } \\
\text { CT-CT }\end{array}$ & $\begin{array}{c}\text { Change Trials } \\
\text { CT-IT }\end{array}$ & & \\
\hline \multirow{2}{*}{ G1 } & RT & $502(69)$ & $723(107)$ & $12.3 *$ & -2.45 & $540(63)$ & $794(119)$ & $17.8^{*}$ & -2.66 \\
\hline & $\mathrm{ACC}$ & $92.5(6.8)$ & $84.6(11.8)$ & $-4.1 *$ & $(-0.77)$ & $92.7(5.6)$ & $26.4(4.9)$ & $-84.8^{*}$ & $(-0.80)$ \\
\hline \multirow{2}{*}{ G3 } & RT & $497(94)$ & $626(88)$ & $10.8 *$ & $(-0.76)$ & $496(76)$ & $665(95)$ & 15.9 * & $(-0.77)$ \\
\hline & $\mathrm{ACC}$ & $92.9(11.2)$ & $95.3(9.6)$ & 1.5 & $0.24(0.12)$ & $96.2(6.7)$ & $31.3(3.1)$ & $-89.8 *$ & $23.83(0.99)$ \\
\hline
\end{tabular}

Note: $\mathrm{G} 1$ = children group; G2 = adolescent group; G3 = adult group. RT = Response Time; IB = Incongruent Block; $\mathrm{MB}=$ Mixed Block; CT-CT = Congruent Trial to Congruent Trial; IT-IT = Incongruent Trial to Incongruent Trial; CT-IT $=$ Congruent Trial to Incongruent Trial; IT-CT $=$ Incongruent Trial to Congruent Trial; ${ }^{*} p<0.001$.

With respect to corollary development, the results obtained from the ANOVA allowed us to verify the effect of the Group in the MB trials with rule change - controlled processing - both in the measures of RT $-\mathrm{F}(2)=35.5, p<0.001, \eta 2=0.37$-and in those of accuracy-F $(2)=18.25, p<0.001, \eta 2=0.23$. Table 7 identifies the groups between which differences in RT and response accuracy are present.

Table 7. Comparison between groups in Mixed Block (MB) trials with rule change.

\begin{tabular}{ccccc}
\hline Comparison between Groups & & G1 $\neq \mathbf{G} 2$ & G2 $\neq$ G3 & G1 $\neq \mathbf{G 3}$ \\
\hline Change trials & RT $^{\mathrm{a}}$ & $p<0.001$ & $\mathrm{~ns}$ & $p<0.001$ \\
IT-CT/CT-IT & ACC $^{\mathrm{b}}$ & $p<0.001$ & $p<0.05$ & $p<0.001$ \\
\hline
\end{tabular}

Note: IT-CT= Incongruent Trials- Congruent Trials; CT-IT= Congruent Trials- Incongruent Trials; G1 = children group; $\mathrm{G} 2$ = adolescent group; $\mathrm{G} 3$ = adult group; $\mathrm{RT}$ = Response Time; $\mathrm{ACC}=$ Accuracy; ns = not significant; post hoc comparison method: ${ }^{\mathrm{a}}$ Bonferroni, ${ }^{\mathrm{b}}$ Games-Howell. 

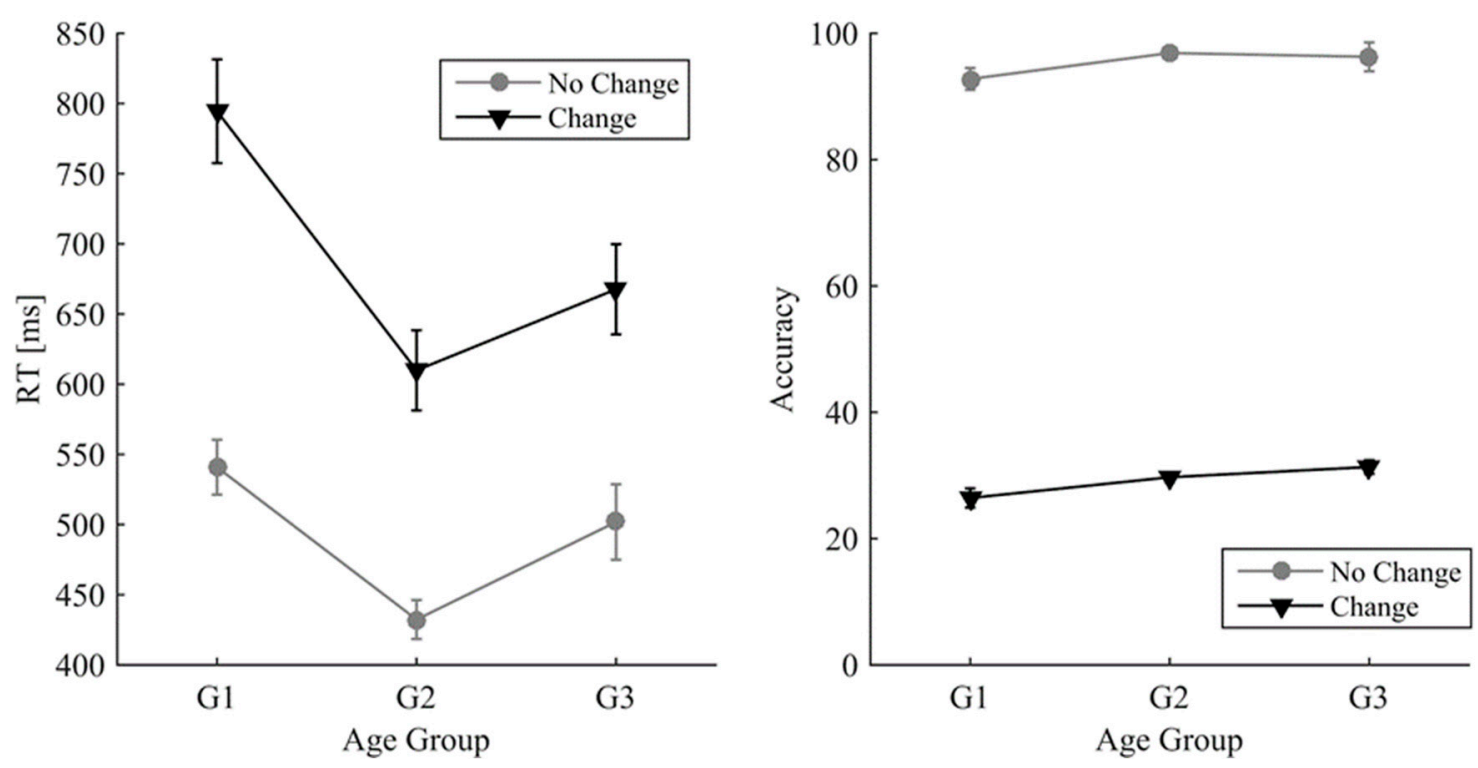

Figure 3. Response Time (RT) and Accuracy among all trials involving some change condition-partial or total-versus repeat trials in the Mixed Block. The bars represent the standard error.

\section{Discussion}

In general, the discriminated analysis of the corollaries made it possible to verify them partially. In this sense, the basic question common to all corollaries was the following: How does the cognitive system work? Is there a global mode-barely discriminate-which activates by default and which in operational terms is characterized by speed and efficiency in its operation? Is there a controlled mode-finer and more discriminate-that implies a greater effort and that in operative terms is manifested in decreased speed and accuracy of response? To answer these questions, the results obtained for each corollary are discussed below.

In relation to corollary 1 (corollary contrast), the results show that the prediction about the activation of a more controlled and discriminated mode for a partial change was only met for the group of adults. In this sense, and contrary to our expectations, a total change resulted more difficult than a partial change in children as well as adolescents. The latter was verified in most of the comparisons made between the different types of change (total change and partial change) in each group (children and adolescents). Thus, all measures obtained in partial change reflect a better performance than those of total change, except for accuracy in DRSS, where no differences were recorded.

Nonetheless, in the adult's group, comparisons made between the different types of change showed that, at this stage of development, partial change is costlier and generates more effort in cognitive terms than total change. However, it should be noted that these results were obtained for one of the partial change indexes-DRMS. When performance was compared between the other partial change index-DRMS—and total change, there were no significant differences.

In summary, the overall reading of the results shows that total change condition demands more discriminated and costly processing than partial change condition, at least for the children and adolescents' groups. However, the situation begins to change in the adult's group, where at least one of the partial change conditions seems to generate greater difficulty and cognitive effort, which is consistent with corollary 1 prediction. In this group, at least one of the partial change conditions generates more controlled and discriminated processing than total change. This is consistent with the results obtained in another study [9] that compares different change conditions in adults; however, an important difference between that research and the present study is that dissimilar developmental stages have been included and compared, which has allowed us to observe that the prediction of the corollaries may or may not be fulfilled depending on the participants' developmental stage. These results differ from those obtained in other studies $[14,15,39]$ where correspondence with 
the corollary was confirmed in children, adolescents, and adults. Additionally, in relation to the comparisons between the two types of partial change (SRDS/DRSS), it is interesting to highlight that no significant differences were found between these conditions, except for the adults, who presented less speed in the DRSS condition compared to the SRDS condition. In most of the comparisons made between these conditions in the other groups, the evidence shows that control and processing demand is similar, since there are no substantial differences in performance.

With respect to the developmental hypothesis (corollary development), a group effect was found in most measures of change (partial change and total change). As change requires the activation of controlled and discriminate processes, the evidence is compatible with findings that establish a systematic and gradual improvement of these processes starting in adolescence $[17,18,40]$. More specifically, the comparisons between groups showed similar performance between adolescents and adults, whereas the children group differed more clearly from these two groups, presenting significantly slower and less accurate responses in almost all measures based on RT.

In summary, in relation to corollary 1, while the evidence makes it possible to confirm the assumption about the development of the controlled mode of operation (corollary development), the same is not true for the assumption about the type of change (corollaries contrast), which was only found in the adult group in the RT of the DRSS condition.

With respect to corollary 2, the evidence shows a worse performance in the conditions in which the irrelevant visual feature (stimulus location) is in conflict with the relevant visual property (stimulus identity); this explains a lower accuracy and a delayed response in IB compared to the condition in which this conflict is absent in CB. In general, these results suggest that the need to ignore the irrelevant visual feature implies a more discriminated and controlled mode of functioning (corollary contrast). However, these differences concern comparisons between the pure congruent block and the pure incongruent block, which exclusively contain congruent or incongruent trials.

When comparisons involve both types of stimuli in the mixed block, the vast majority of differences tend to disappear. This result does not seem to contradict the corollary's main assumption, since although the stimuli are the same (congruent versus incongruent), the context in which they are presented is different. The fundamental difference between pure and mixed blocks is that while in the latter the participant must quickly and accurately execute rule and response site changes, the pure blocks only require a response site change. Consequently, the difficulty in the MB seems to respond not so much to the type of stimulus to which the participant must respond, but rather to the requirement of making accurate and rapid changes. As explained in other studies $[9,17]$, and in terms of executive processes, this block requires the activation of two rules in working memory and the ability to switch between alternative responses (cognitive flexibility), requirements absent in the pure blocks. Considering this distinction, the results obtained confirm the main assumption posited in the corollary.

With respect to the developmental hypothesis about the controlled mode (corollary development), in general a group effect was found in the different measures we analyzed (incongruent block and incongruent stimuli of the MB). However, looking at the RT of the incongruent block, the best performance was observed in the adolescent group and, surprisingly, the adults presented a similar performance to that of the children. This underscores the importance of deepening the analysis of this data and the need to undertake new studies aimed at comparing the controlled mode among adolescents and adults, but including more limited or shorter age intervals that would allow for the analysis of differences in detail.

Additionally, when the incongruent stimuli of the MB are taken as a measure of the controlled mode, the results show a similar pattern, with adolescents performing faster than adults and children. Adults, in turn, respond more quickly than children to these types of stimuli. On the other hand, no differences were identified in the accuracy of response between adults and adolescents.

Although different studies have reported age-related changes $[17,41]$, the changes found in this study present a different pattern than expected, since, as a whole, adolescents displayed better 
performance than adults (corollary development). In summary, the data provided here confirms the prediction presented in the corollary (corollary contrast), since, regardless of age, when the trials included irrelevant visual features (stimulus location) as in the case of incongruent stimuli, there was a decrease in performance, which can be interpreted as the result of inhibiting the irrelevant visual properties, thus implying a more discriminated and controlled functioning compared to the conditions in which there is no conflict between location (irrelevant property) and stimulus identity (congruent trials). On the other hand, the evidence also confirms the existence of changes associated with age, which implies the presence of a developmental effect (corollary development). However, the development path does not seem to follow a linear pattern (Tables 4 and 5). Hence the need to carry out studies that consider several stages of development along the life course [10], as they would allow to analyze the possibility of the existence of U-shaped trajectories for some of the processes that demand a more controlled and discriminated processing [42].

Finally, in relation to corollary 3, the data obtained clearly show that it is easier to inhibit always than to do so only some of the time (corollary contrast). In this way, the age group analyses show a decrease in response speed in the MB. This block requires inhibition only in some of the trials, compared to the incongruent block, which requires continuous activation of the inhibitory process. In turn, the speed and accuracy of response decreased substantially in mixed block trials that involved a rule change. In this condition, although the repetition of the rule never exceeded four consecutive trials, its permanence-regardless of the type of rule in question-clearly favored performance, while change substantially impaired it. Therefore, based on the evidence we can infer that the requirement of change involves a type of controlled processing that manifests itself immediately in a marked worsening of performance. This performance pattern is compatible with the results described in Section 1 [17] and by those obtained in other more recent studies that focus on other stages of development $[9,29]$.

With regard to the developmental hypothesis (corollary development), there is a pattern similar to that of corollary 2. A group effect was found in the trials that involved a rule change in the mixed block, that is, those involving a controlled mode of functioning. However, the change was different than expected, as adolescents performed significantly better than adults, who in turn performed better than children. Further, the detailed analysis of corollary 1 regarding the different types of change shows a clear group effect that includes both total and partial change (see corollary development, corollary 1). As a result, the evidence is compatible with a large number of studies that show that change-or cognitive flexibility—requires effort and control, and improves with age [17,18,29].

Nonetheless, it would be interesting to carry out a larger number of studies on development of cognitive control in general, and of cognitive flexibility in particular, in order to explain one of the most striking findings of this study: the better performance of adolescents compared to young adults. Besides, it is possible that the wide age range that characterizes the adolescents group hampers the interpretation of our results. The inclusion of more homogeneous age groups (e.g., early adolescence, late adolescence) should be considered for future studies.

On the other hand, and in relation to the task used, the possibility for participants to reduce the two rules into one- "pressing in direction to the site where the finger is pointing"- thus eliminating the rule change related to the mixed block, could be argued. We suppose that if that had been the case, we should have found a better performance in the adult group instead of the adolescent group, due to a higher language domain as a self-regulatory mechanism. In this sense, similar tasks such as the Dots Test [17] would allow to avoid this simplification due to the absence of visual cues indicating the response site. Nonetheless, that type of task presents a high working memory requirement, as participants must activate the two rules arbitrarily associated with the stimuli, thus complicating the task and including the participation of another executive process. 


\section{Conclusions}

The present study provided relevant evidence about the existence of two differentiable cognitive system processing ways (i.e., a comprehensive and least discriminated mode and a controlled mode requiring greater precision or discrimination). Our findings and argumentations go along with different classical theoretical development approaches in cognitive science that assume this distinction, although using other terms $[3,4,8]$. Our findings related to the analysis of the three corollaries suggested that under conditions that require control and discrimination, children, adolescents, and adults presented a control processing cost that is traduced in response accuracy and speed and that indicates the activation of a controlled and more discriminated cognitive system processing mode.

In summary, the collective analysis of the three corollaries shows that under conditions that require control and discrimination, children, adolescents, and adults presented a cost that manifested itself in response accuracy and speed. Thus, the interest in the detailed study of each corollary lies in the attempt to explain the way people respond under conditions that require cognitive effort. Hence, each corollary involves high-level cognitive functions that allow for the regulation of thought, action, and emotion in order to achieve goals $[43,44]$. The importance of these cognitive functions can be observed both in academic achievement and social competence, as well as in everyday tasks such as reading and driving $[45,46]$. For this reason, the study of the controlled mode of functioning is central to cognitive science.

Another finding of interest is that the controlled and discriminated cognitive system processing mode in adolescents tends to be more accurate and efficient than that of adults and children. In relation to this issue, most studies suggest that there is a linear improvement that occurs from childhood, that it continues to improve progressively during adolescence and that it tends to stabilize and maintain beyond young adulthood [17]. In this sense, our result did not support this idea because we found a better RT and ACC performance in adolescence. However, in order to deepen this discussion, in further research it would be desirable to conduct complementary studies that would include smaller age ranges in order to determine exactly the point on the developmental timeline in which adulthood begins to show a decrease in the controlled mode of processing. As mentioned in the introduction, adolescence is now considered a sensitive and a central period of development, and in this regard it is essential to have more research for analyzing the typical changes associated with this crucial developmental stage.

To conclude, it is interesting to note that these results, although it was not the purpose of the study, provide empirical evidence validity of construct to the Fingers Task. Thus, in a general way, the most controlled and discriminated mode of operating the cognitive system was activated in those conditions that, according to the theory, demanded this type of processing. On one side, this was replicated in the different age groups. Therefore, this finding is promising insofar as it encourages a more in-depth study of this procedure for the evaluation of poorly controlled cognitive processes, as well as more controlled and discriminated processes. On the other hand, we think that in further research it will be necessary to consider the analysis of the corollaries through their contrast in older adults because this developmental stage is not yet deeply explored in this area of study.

Author Contributions: Conceptualization, I.M.I., M.M.R., M.F.L.-R. and E.N.-P.; Writing-original draft preparation, I.M.I., M.M.R., A.G.-C. and M.F.L.-R.; Writing-review and editing, E.N.-P.; Software, A.G.-C., L.C.-J., Y.A., M.L.A. and F.S.; Data curation, M.L.A. and F.S.; Methodology, M.M.R., E.V.Z., M.F.L.-R. and E.N.-P.; Visualization, M.M.R., E.V.Z. and Y.A.; Project administration, I.M.I., M.M.R. and L.C.-J.; Funding acquisition, I.M.I., M.F.L.-R. and E.N.-P. All authors have read and agreed to the published version of the manuscript.

Funding: This research was funded by CONICET (PIP 2015-2017: 11220150100480CO).

Conflicts of Interest: The authors declare no conflict of interest.

\section{References}

1. Daouia, A.; Girard, S.; Stupfler, G. Tail expectile process and risk assessment. Bernoulli 2020, 26, 531-556. [CrossRef] 
2. Diamond, A. All or none hypothesis: A global-default mode that characterizes the brain and mind. Dev. Psychol. 2009, 45, 130-138. [CrossRef] [PubMed]

3. Frankish, K. Dual-Process and Dual-System Theories of Reasoning. Philos. Compass 2010, 5, 914-926. [CrossRef]

4. Saab, S. Modos de autoengaño y de razonamiento: Teorías de proceso dual. Anal. Filos. 2011, 31, $193-218$.

5. Blakemore, S.-J.; Frith, U. The learning brain: Lessons for education: A precis. Dev. Sci. 2005, 8, 459-465. [CrossRef]

6. Camacho, M.C.; Quiñones-Camacho, L.E.; Perlman, S.B. Does the child brain rest? An examination and interpretation of resting cognition in developmental cognitive neuroscience. NeuroImage 2020, 212, 116688. [CrossRef]

7. Ramsey, R.; Ward, R. Challenges and opportunities for top-down modulation research in cognitive psychology. Acta Psychol. 2020, 209, 103118. [CrossRef]

8. Nigg, J.T. Annual Research Review: On the relations among self-regulation, self-control, executive functioning, effortful control, cognitive control, impulsivity, risk-taking, and inhibition for developmental psychopathology. J. Child Psychol. Psychiatry 2017, 58, 361-383. [CrossRef]

9. Introzzi, I.M.; Richard's, M.M.; Comesaña, A.; Coni, A.G. Cognitive functioning: Is it all or none? Psychol. Res. 2018, 83, 1137-1146. [CrossRef]

10. Poon, K. Hot and Cool Executive Functions in Adolescence: Development and Contributions to Important Developmental Outcomes. Front. Psychol. 2018, 8, 2311. [CrossRef]

11. Ruotsalainen, I.; Gorbach, T.; Perkola, J.; Renvall, V.; Syväoja, H.J.; Tammelin, T.H.; Karvanen, J.; Parviainen, T. Physical activity, aerobic fitness, and brain white matter: Their role for executive functions in adolescence. Dev. Cogn. Neurosci. 2020, 42, 100765. [CrossRef] [PubMed]

12. Bodison, S.C.; Colby, J.B.; Sowell, E.R. Structural brain development: Birth through adolescence. In Neural Circuit and Cognitive Development; Academic Press: Cambridge, MA, USA, 2020; pp. 289-317. [CrossRef]

13. Blakemore, S.J.; Choudhury, S. Development of the adolescent brain: Implications for executive function and social cognition. J. Child Psychol. Psychiatry 2006, 47, 296-312. [CrossRef] [PubMed]

14. Kleinsorge, T. Response repetition benefits and costs. Acta Psychol. 1999, 103, 295-310. [CrossRef]

15. Rogers, R.D.; Monsell, S. Costs of a predictable switch between simple cognitive tasks. J. Exp. Psychol. Gen. 1995, 124, 207-231. [CrossRef]

16. Schurz, M.; Maliske, L.; Kanske, P. Cross-network interactions in social cognition: A review of findings on task related brain activation and connectivity. Cortex 2020, 130, 142-157. [CrossRef]

17. Davidson, M.C.; Amso, D.; Anderson, L.C.; Diamond, A. Development of cognitive control and executive functions from 4 to 13 years: Evidence from manipulations of memory, inhibition, and task switching. Neuropsychologia 2006, 44, 2037-2078. [CrossRef]

18. Zelazo, P.D.; Craik, F.I.M.; Booth, L. Executive function across the life span. Acta Psychol. 2004, 115, 167-183. [CrossRef]

19. Hommel, B.; Proctor, R.W.; Vu, K.-P.L. A feature-integration account of sequential effects in the Simon task. Psychol. Res. 2004, 68, 1-17. [CrossRef]

20. Lu, C.-H.; Proctor, R.W. The influence of irrelevant location information on performance: A review of the Simon and spatial Stroop effects. Psychon. Bull. Rev. 1995, 2, 174-207. [CrossRef]

21. Simon, H. Invariants of human behavior. Annu. Rev. Psychol. 1990, 41, 1-19. [CrossRef]

22. Verwey, W.B.; Wright, D.L.; Van Der Lubbe, R.H. The Simon effect in a discrete sequence production task: Key-specific stimuli cannot be ignored due to attentional capture. Acta Psychol. 2020, 205, 103044. [CrossRef] [PubMed]

23. Kornblum, S.; Lee, J.W. Stimulus-response compatibility with relevant and irrelevant dimensions that do and do not overlap with the response. J. Exp. Psychol. Hum. Percept. Perform. 1995, 21, 855-875. [CrossRef] [PubMed]

24. Savic, O.; Thierry, G.; Kovic, V. Conceptual relation preference: A matter of strategy or one of salience? Acta Psychol. 2020, 204, 103018. [CrossRef] [PubMed]

25. Gerardi-Caulton, G. Sensitivity to spatial conflict and the development of self-regulation in children 24-36 months of age. Dev. Sci. 2000, 3, 397-404. [CrossRef] 
26. Band, G.P.H.; Van Der Molen, M.W.; Overtoom, C.C.; Verbaten, M.N. The Ability to Activate and Inhibit Speeded Responses: Separate Developmental Trends. J. Exp. Child Psychol. 2000, 75, 263-290. [CrossRef] [PubMed]

27. Kirkham, N.Z.; Cruess, L.; Diamond, A. Helping children apply their knowledge to their behavior on a dimension-switching task. Dev. Sci. 2003, 6, 449-467. [CrossRef]

28. Waszak, F.; Hommel, B.; Allport, A. Task-switching and long-term priming: Role of episodic stimulus-task bindings in task-shift costs. Cogn. Psychol. 2003, 46, 361-413. [CrossRef]

29. Richard'S, M.M.; Krzemien, D.; Valentina, V.; Vernucci, S.; Zamora, E.; Comesaña, A.; Coni, A.G.; Introzzi, I. Cognitive flexibility in adulthood and advanced age: Evidence of internal and external validity. Appl. Neuropsychol. Adult 2019,1-15. [CrossRef]

30. Richards, M.M.; Vernucci, S.; Stelzer, F.; Introzzi, I.; Guàrdia-Olmos, J. Exploratory data analysis of executive functions in children: A new assessment battery. Curr. Psychol. 2018, 39, 1610-1617. [CrossRef]

31. Hollingshead, A.B. Four Factor Index of Social Status. Yale J. Soc. 2011, 8, 21-51.

32. Introzzi, I.; Canet Juric, L. TAC: Tareas de Autorregulación Cognitiva [Software]. Application for Deposit in Custody of Unpublished Work in the National Direction of Copyright. 2019. Available online: https: //tac.com.ar/evaluacion/ (accessed on 9 October 2020).

33. Monsell, S. Task switching. Trends Cogn. Sci. 2003, 7, 134-140. [CrossRef]

34. Wylie, G.; Allport, A. Task switching and the measurement of "switch costs". Psychol. Res. 2000, 63, $212-233$. [CrossRef] [PubMed]

35. National Council for Scientific and Technical Research of Argentina (CONICET). Guidelines for Ethical Behavior in the Social Sciences and Humanities. Resolution of the Board of Directors of the CONICET. Available online: https://www.conicet.gov.ar/wp-content/uploads/RD-20061211-2857.pdf (accessed on 9 October 2020).

36. American Psychological Association (APA). Ethical Principles of Psychologists and Code of Conduct. Washington, DC. Available online: http://www.apa.org/ethics/code/ethics-code-2017.pdf (accessed on 9 October 2020).

37. World Medical Association (WMA). Declaration of Helsinki-Ethical Principles for Medical Research Involving Human Subjects. Available online: https://www.wma.net/policies-post/wma-declaration-ofhelsinki-ethical-principles-for-medical-research-involving-human-subjects/ (accessed on 9 October 2020).

38. Kreutzer, J.S.; Caplan, B.; DeLuca, J. Encyclopedia of Clinical Neuropsychology; Springer: New York, NY, USA, 2011.

39. Meiran, N. Modeling cognitive control in task-switching. Psychol. Res. 2000, 63, 234-249. [CrossRef] [PubMed]

40. Huizinga, M.; Dolan, C.V.; Van Der Molen, M.W. Age-related change in executive function: Developmental trends and a latent variable analysis. Neuropsychologia 2006, 44, 2017-2036. [CrossRef] [PubMed]

41. Zamora, E.; Vernucci, S.; Del Valle, M.; Introzzi, I.; Richard'S, M.M. Assessing cognitive inhibition in emotional and neutral contexts in children. Educ. Dev. Psychol. 2020, 37, 56-66. [CrossRef]

42. Pauls, F.; Macha, T.; Petermann, F. U-Shaped Development: An Old but Unsolved Problem. Front. Psychol. 2013, 4, 301. [CrossRef] [PubMed]

43. Chevalier, N.; Wiebe, S.A.; Huber, K.L.; Espy, K.A. Switch detection in preschoolers' cognitive flexibility. J. Exp. Child Psychol. 2011, 109, 353-370. [CrossRef]

44. Friedman, N.P.; Miyake, A. Unity and diversity of executive functions: Individual differences as a window on cognitive structure. Cortex 2017, 86, 186-204. [CrossRef]

45. Bull, R.; Espy, K.A.; Wiebe, S.A.; Sheffield, T.D.; Nelson, J.M. Using confirmatory factor analysis to understand executive control in preschool children: Sources of variation in emergent mathematic achievement. Dev. Sci. 2011, 14, 679-692. [CrossRef]

46. Chevalier, N.; Huber, K.L.; Wiebe, S.A.; Espy, K.A. Qualitative change in executive control during childhood and adulthood. Cognition 2013, 128, 1-12. [CrossRef]

Publisher's Note: MDPI stays neutral with regard to jurisdictional claims in published maps and institutional affiliations.

(C) 2020 by the authors. Licensee MDPI, Basel, Switzerland. This article is an open access article distributed under the terms and conditions of the Creative Commons Attribution (CC BY) license (http://creativecommons.org/licenses/by/4.0/). 